\title{
Gevrey regularity and analyticity for the modified Camassa-Holm equation
}

\section{Qingyu Zhou ${ }^{1} \cdot$ Xiaolin Pan $^{1} \cdot$ Shouming Zhou ${ }^{1}$}

Received: 28 July 2021 / Accepted: 23 January 2022 / Published online: 21 February 2022

(C) The Author(s) 2022

\begin{abstract}
This paper deals with the analyticity and Gevrey regularity for the Cauchy problem of a modified Camassa-Holm $(\mathrm{mCH})$ equation in Sobolev-Gevrey space $G_{r, s}^{\delta}$ with $s>\frac{1}{2}, r \geq 1$ and $0<\delta<1$, and a lower bound of lifespan and continuity of the datato-solution map was also obtained.
\end{abstract}

Keywords Camassa-Holm equation · Analyticity · Gevrey regularity

Mathematics Subject Classification 35Q53 $\cdot 35 \mathrm{~B} 30 \cdot 35 \mathrm{~B} 44 \cdot 35 \mathrm{C} 07 \cdot 35 \mathrm{G} 25$

\section{Introduction}

In this paper, we consider the following modified Camassa-Holm equation ( $\mathrm{mCH})$

$$
\begin{cases}\gamma_{t}=\frac{\gamma^{2}}{2}+\lambda v-(v \gamma)_{x}, & t \geq 0, \quad x \in \mathbb{R}, \\ v_{x x}-v=\gamma_{x}+\frac{\gamma^{2}}{2 \lambda}, & t>0, \quad x \in \mathbb{R}, \\ \gamma(x, 0)=\gamma_{0}(x), & t=0, x \in \mathbb{R} .\end{cases}
$$

It is known that the Camassa-Holm equation describes pseudo-spherical surfaces and therefore its integrability properties can be studied by geometrical means [21], using this fact, Gorka and Reyes (see (6.1)-(6.2) in [15]) introduced a "Miura transform" and proposed the modified Camassa-Holm (1.1) equation, in analogy with the Korteweg-de Vries theory. They also obtained conservation laws for (1.1) and shown that it describes pseudo-spherical surfaces, so that, in particular, it is the integrability condition of an $\operatorname{sl}(2, \mathbb{R})$-valued linear problem (cf. $[15,16])$.

Xiaolin Pan

20130309@cqnu.edu.cn

Qingyu Zhou

zqy499627738@126.com

1 College of Mathematics Science, Chongqing Normal University, Chongqing 401331, China 
The original Camassa-Holm equation was obtained by Fuchssteiner and Fokas by using the method of recursion operators [14],

$$
m_{t}+2 u_{x} m+u m_{x}=0, m=\left(1-\partial_{x}^{2}\right) u
$$

and later derived as a model for unidirectional propagation of shallow water over a flat bottom by Camassa and Holm [1]. Analogous to the famous $\mathrm{KdV}$ equation, the Camassa-Holm equation (1.2) also has a bi-Hamiltonian structure [1, 14], which is completely integrable not only in the sense of the existence of a Lax pair [1], but also (by means of inverse scattering and inverse spectral theory) as an infinitedimensional Hamiltonian flow that can be linearised in suitable action-angle variables, cf. (see $[3,4,8,11,12]$ ). The orbital stability of solitary waves and the stability of the peakons $(k=0)$ for the Camassa-Holm equation are investigated by Constantin and Strauss $[9,10]$. The advantage of the Comassa-Holm equation in comparison with the famous $\mathrm{KdV}$ equation lies in the fact that the Camassa-Holm models the peculiar wave breaking phenomena, that is, the solution remains bounded but its slope becomes unbounded in finite time (cf. [2, 7]). In addition to wave breaking, one of the most interesting aspects of the equation is the existence of peakon solutions, namely, the travelling wave solutions of greatest height of the governing equations for water waves have a peak at their crest (cf. $[5,6])$. The latest progress about the Camassa-Holm type equations can be refer to [20] and references therein.

Recently, Luo, Qiao and Yin [19] established the local well-posedness for the Cauchy problem of the Equ. (1.1) in nonhomogeneous Besov spaces by LittlewoodPaley theory and transport equation theory. In this paper, we show the Geverey and analytic regularity of the Cauchy problem for $\mathrm{mCH}$ system. We first introduce the Sobolev-Gevrey spaces (cf. [13]).

Definition 1.1 Let $\mathrm{s}$ be a real number and $r, \delta>0$. A function $f \in G_{r, s}^{\delta}(\mathbb{R})$ if and only if $f \in \mathcal{C}^{\infty}(\mathbb{R})$ and satisfies

$$
\|f\|_{G_{r, s}^{\delta}(\mathbb{R})}=\left(\int_{\mathbb{R}}\left(1+|\xi|^{2}\right)^{s} e^{2 \delta|\xi|^{\frac{1}{r}}}|\hat{f}(\xi)|^{2} d \xi\right)^{\frac{1}{2}}<\infty .
$$

Note that the Fourier multiplier $e^{\delta(-\Delta)^{\frac{1}{2 r}}}$ by: $e^{\delta(-\Delta)^{\frac{1}{2 r}}} f=\mathscr{F}^{-1}\left(e^{\delta|\xi|^{\frac{1}{r}}} \hat{f}\right)$, which implies that $\|f\|_{G_{r, s}^{\delta}(\mathbb{R})}=\left\|e^{\delta(-\Delta)^{\frac{1}{2 r}}} f\right\|_{H^{s}(\mathbb{R})}$. For $0<r<1$, it is called ultra-analytic function. If $r=1$, it is usual analytic function and $\delta$ is called the radius of analyticity. If $r>1$, it is the Gevrey class function.

It is well known that the solutions to the Camassa-Holm type systems are analytic in both space and time variables for a short time (see [17]). However, it gives no estimate about the size of the analytic lifespan $\delta$. Also, it dose not supply information about the evolution of the uniform radius of analyticity. Recently, Barostichi et al. solved these important problems for Comassa-Holm and other nonlocal equations and systems by using an Ovsyannikov type theorem for an autonomous abstract Cauchy problem in a scale of decreasing Banach spaces, and they also studied the stability of their solution map. By taking the advantage of this idea, Luo and Yin [18] proved the Gevrey regularity and analyticity of these systems be a generalized 
Ovsyannikov theorem. Our motivation to solve this problem follow the line of [17, 18].

Theorem 1.1 Let $\gamma_{0} \in G_{r, s}^{1}(\mathbb{R})$ with $r \geq 1$ and $s>1 / 2$. Thus, for all $0<\delta<1$, there exists a $T_{0}>0$ such that the $m C H$ system has a unique solution $\gamma$ which is holomorphic in $|t|<D_{r} \frac{(1-\delta)^{r} T_{0}}{2^{r+1}}$ with values in $G_{r, s}^{\delta}(\mathbb{R})$, where $D_{r}=\frac{1}{2^{r}-2+\frac{1}{2^{r+1}}}$. Moreover $T_{0}=\frac{1}{C_{s} 2^{2 r+7}\left(e^{-r} r^{r}+2\right)\left[\left(\left\|\gamma_{0}\right\|_{G_{r, s}^{1}(\mathbb{R})}+\frac{1}{2}\right)\left\|\gamma_{0}\right\|_{G_{r, s}^{1}(\mathbb{R})}+\frac{1}{12}\right]}$.

With the existence and uniqueness at hand, we next show the continuity of the data-to-solution map in $G_{r, s}^{1}(\mathbb{R})$, which means that:

Definition 1.2 If for a given $\gamma_{0}^{\infty} \in G_{r, s}^{1}(\mathbb{R})$ with $r \geq 1$ and $s>\frac{1}{2}$, there exists a $T\left(\left\|\gamma_{0}^{\infty}\right\|_{G_{r, s}^{1}(\mathbb{R})}\right)$ such that for any sequence $\gamma_{0}^{n} \in G_{r, s}^{1}(\mathbb{R})$, and $\left\|\gamma_{0}^{n}-\gamma_{0}^{\infty}\right\|_{G_{r, s}^{1}(\mathbb{R})} \rightarrow 0$ for $n \rightarrow \infty$, the corresponding solution $\gamma^{n}(t)$ of $\mathrm{mCH}$ system satisfies $\left\|\gamma^{n}(t)-\gamma^{\infty}(t)\right\|_{E_{T}} \longrightarrow 0$ for $n \rightarrow \infty$, where

$$
\|\gamma\|_{E_{T}}=\sup _{|t|<D_{r} \frac{T(1-\delta)^{r}}{2^{r+1}}, 0<\delta<1}\left(\|\gamma\|_{G_{r, s}^{\delta}(\mathbb{R})}(1-\delta)^{r} \sqrt{1-\frac{|t|}{T(1-\delta)^{r}}}\right) .
$$

Our last result about the continuity of the data-to-solution map was stated as follows:

Theorem 1.2 If $\gamma_{0} \in G_{r, s}^{1}(\mathbb{R})$ with $r \geq 1$ and $s>\frac{1}{2}$. Then the data-to-solution map $\gamma_{0} \rightarrow \gamma$ of the Equ. (1.1) is continuous from $G_{r, s}^{1}(\mathbb{R})^{2}$ into the solutions space.

Remark 1 In the period case, the Sobolev-Gevrey norm can be defined as follows

$$
\|f\|_{G_{r, s}^{\delta}(\mathbb{T})}=\left(\sum_{k \in \mathbb{Z}}\left(1+|k|^{2}\right)^{s} e^{2 \delta|k|^{\frac{1}{r}}}|\hat{f}(k)|^{2}\right)^{\frac{1}{2}}=\left\|e^{\delta(-\Delta)^{\frac{1}{2 r}}} f\right\|_{H^{s}(\mathbb{T})} .
$$

in view of the same argument as in Theorems 1.1 and 1.2, we can get the similar Gevrey regularity and analytic for the $\mathrm{mCH}(1.1)$.

The plan of this paper is organized as follows. In the next section, we recall some properties about Sobolev-Gevrey spaces. In the last section, we prove the analyticity and Gevrey regularity of the solutions to the modified Camassa-Holm equation, and prove Theorems 1.1-1.2. 


\section{Preliminaries}

In this section, we will investigate the analytic solutions to $\mathrm{mCH}$ system (1.1) by nonlinear Cauchy-Kowalevski theorem, and the Cauchy-Kowalevski theorem is read as follows:

Theorem 2.1 (See Theorem 3.1 in $[18,22]$ ) Let $\left(X_{\delta},\|\cdot\|_{\delta}\right)_{0<\delta \leq 1}$ be a scale of decreasing Banach spaces, such that for any $0<\delta^{\prime} \leq 1$ we have $X_{\delta} \subset X_{\delta^{\prime}}$ with $\|\cdot\|_{\delta^{\prime}}<\|\cdot\|_{\delta}$. Consider the Cauchy problem

$$
\left\{\begin{array}{l}
\frac{d \gamma}{d t}=F(t, \gamma(t)), \\
\gamma(0)=0 .
\end{array}\right.
$$

Let $T, R>0$ and $r \geq 1$. For given $\gamma_{0} \in X_{1}$, suppose that $F$ satisfies the following conditions:

(1) If for any $0<\delta^{\prime}<\delta<1$, the function $t \mapsto \gamma(t)$ is holomorphic on $|t|<T$ and continuous on $|t| \leq T$ with values in $X_{\delta}$ and

$$
\sup _{|t| \leq T}\|\gamma(t)\|_{\delta}<R
$$

then $t \mapsto F(t, \gamma(t))$ is a holomorphic function on $|t|<T$ with values in $X_{\delta}$.

(2) For any $0<\delta^{\prime}<\delta<1$ and $\gamma_{1}, \gamma_{2} \in \overline{B\left(\gamma_{0}, R\right)} \subset X_{\delta}$, that is, $\left\|\gamma_{1}\right\|_{\delta}<R$, $\left\|\gamma_{2}\right\|_{\delta}<R$, there exits a positive constant $L$ depending on $\gamma_{0}$ and $R$ such that

$$
\sup _{|t| \leq T}\left\|F\left(t, \gamma_{1}\right)-F\left(t, \gamma_{2}\right)\right\|_{\delta^{\prime}} \leq \frac{L}{\left(\delta-\delta^{\prime}\right)^{r}}\left\|\gamma_{1}-\gamma_{2}\right\|_{\delta}
$$

(3) There exists a $M>0$ depending on $\gamma_{0}$ and $R$ such that for any $0<\delta<1$,

$$
\sup _{|t| \leq T}\left\|F\left(t, \gamma_{0}\right)\right\|_{\delta} \leq \frac{M}{(1-\delta)^{r}} .
$$

Then there exists a $T_{0} \in(0, T)$ and a unique function $\gamma(t)$ to the Cauchy problem (2.3), which is holomorphic in $|t|<D_{r} \frac{(1-\delta)^{r} T_{0}}{2^{r+1}}$ with value in $X_{\delta}$ for every $\delta \in(0,1)$

Remark 2.1 $T_{0}=\min \left\{\frac{1}{2^{2 r+4} L}, \frac{2^{r+1} R}{2^{r+1} 2^{2 r+3} L R+M D_{r}}\right\}, D_{r}=\frac{1}{2^{r}-2+\frac{1}{2^{r+1}}}$. (see Lemma 3.7 in $[18,22])$.

If $X_{\delta}=G_{r, s}^{\delta}(\mathbb{R})$ (see Definition 1.1), then $G_{r, s}^{\delta}(\mathbb{R}) \hookrightarrow G_{r, s}^{\delta^{\prime}}(\mathbb{R})$ with $0<\delta^{\prime}<\delta$, $G_{r^{\prime}, s}^{\delta}(\mathbb{R}) \hookrightarrow G_{r, s}^{\delta}(\mathbb{R})$ with $0<r^{\prime}<r, G_{r, s}^{\delta}(\mathbb{R}) \hookrightarrow G_{r, s^{\prime}}^{\delta}(\mathbb{R})$ with $s^{\prime}<s$. Moreover, $G_{r, s}^{\delta}(\mathbb{R})$ has the following three properties: 
Proposition 2.1 (See Proposition 2.5-2.6 in [18]) Let $s>1 / 2, r \geq 1$ and $\delta>0$, then $G_{r, s}^{\delta}(\mathbb{R})$ is an algebra, and for any $\gamma_{1} \in G_{r, s-1}^{\delta}(\mathbb{R}), \gamma_{2} \in G_{r, s}^{\delta}(\mathbb{R})$, there is a constant $C_{s}$ that is only dependent of s such that

$$
\left\|\gamma_{1} \gamma_{2}\right\|_{G_{r, s-1}^{\delta}(\mathbb{R})} \leq C_{s}\left\|\gamma_{1}\right\|_{G_{r, s-1}^{\delta}(\mathbb{R})}\left\|\gamma_{2}\right\|_{G_{r, s}^{\delta}(\mathbb{R})} .
$$

Moreover, for any $\gamma_{1}, \gamma_{2} \in G_{r, s}^{\delta}(\mathbb{R})$, there is a constant $C_{s}^{\prime}$ that is only dependent of $s$ such that

$$
\left\|\gamma_{1} \gamma_{2}\right\|_{G_{r, s}^{\delta}(\mathbb{R})} \leq C_{s}^{\prime}\left\|\gamma_{1}\right\|_{G_{r, s}^{\delta}(\mathbb{R})}\left\|\gamma_{2}\right\|_{G_{r, s}^{\delta}(\mathbb{R})}
$$

Proposition 2.2 (See Proposition 2.4 in [18]) Let $s$ be a real number and $r>0$, $s>0$. For any $0<\delta^{\prime}<\delta$, we have

$$
\left\|\gamma_{x}\right\|_{G_{r, s-1}^{\delta}(\mathbb{R})} \leq\|\gamma\|_{G_{r, s}^{\delta}(\mathbb{R})}, \quad\left\|\gamma_{x}\right\|_{G_{r, s}^{\delta^{\prime}}(\mathbb{R})}<\frac{e^{-r} r^{r}}{\left(\delta-\delta^{\prime}\right)^{r}}\|\gamma\|_{G_{r, s}^{\delta}(\mathbb{R})} .
$$

Proposition 2.3 (See Proposition 2.7 in [18]) For any $s \in \mathbb{R}, r, \delta>0$ and $\gamma \in G_{r, s}^{\delta}(\mathbb{R})$, we get

$$
\left\|\Lambda^{-2} \gamma\right\|_{G_{r, s}^{\delta}(\mathbb{R})} \leq\|\gamma\|_{G_{r, s}^{\delta}(\mathbb{R})},\left\|\Lambda^{-2} \partial_{x} \gamma\right\|_{G_{r, s}^{\delta}(\mathbb{R})} \leq\|\gamma\|_{G_{r, s-1}^{\delta}(\mathbb{R})},
$$

where $\Lambda=\left(1-\partial_{x}^{2}\right)^{\frac{1}{2}}$.

\section{Proof of Theorem 1.1 and 1.2}

In this section, we will prove Theorem 1.1 and 1.2 by Theorem 2.1 and Proposition 2.1, 2.2 and 2.3.

Proof of Theorem 1.1 In order to applying the Theorem 2.1, we rewrite $(\mathrm{mCH})$ as follows:

$$
\left\{\begin{array}{l}
\gamma_{t}=F(\gamma) \doteq \frac{\gamma^{2}}{2}+\lambda v-v_{x} \gamma-v \gamma_{x} \\
v=-\Lambda^{-2}\left(\gamma_{x}+\frac{\gamma^{2}}{2 \lambda}\right) \\
\left.\gamma\right|_{t=0}=\gamma_{0} .
\end{array}\right.
$$

Next, we can prove that function $F(\gamma)$ satisfies the three conditions in Theorem 2.1. According to Propositions 2.1, 2.2 and 2.3, for $0<\delta^{\prime}<\delta, s>\frac{1}{2}$, we can deduce that 


$$
\begin{aligned}
& \|F(\gamma)\|_{G_{r, s}^{\delta^{\prime}}(\mathbb{R})} \leq \frac{1}{2}\left\|\gamma^{2}\right\|_{G_{r, s}^{\delta^{\prime}}(\mathbb{R})}+\|\lambda v\|_{G_{r, s}^{\delta^{\prime}}(\mathbb{R})}+\left\|v_{x} \gamma\right\|_{G_{r, s}^{\delta^{\prime}}(\mathbb{R})}+\left\|v \gamma_{x}\right\|_{G_{r, s}^{\delta^{\prime}}(\mathbb{R})} \\
& \leq \frac{C_{s}}{2}\|\gamma\|_{G_{r, s}^{\delta}(\mathbb{R})}^{2}+C_{s}\|v\|_{G_{r, s}^{\delta}(\mathbb{R})} \\
& \quad+C_{s}\left\|v_{x}\right\|_{G_{r, s}^{\delta^{\prime}}(\mathbb{R})}\|\gamma\|_{G_{r, s}^{\delta^{\prime}}(\mathbb{R})}+C_{s}\|v\|_{G_{r, s}^{\delta^{\prime}(\mathbb{R})}}\left\|\gamma_{x}\right\|_{G_{r, s}^{\delta^{\prime}(\mathbb{R})}} \\
& \leq C_{s}\|\gamma\|_{G_{r, s}^{\delta}(\mathbb{R})}^{2}+\frac{C_{s}}{2}\|\gamma\|_{G_{r, s}^{\delta}(\mathbb{R})}+C_{s}\left\|v_{x}\right\|_{G_{r, s}^{\delta^{\prime}}(\mathbb{R})}\|\gamma\|_{G_{r, s}^{\delta^{\prime}}(\mathbb{R})} \\
& \quad+\frac{C_{s} e^{-r} r^{r}}{\left(\delta-\delta^{\prime}\right)^{r}}\|v\|_{G_{r, s}^{\delta}(\mathbb{R})}\|\gamma\|_{G_{r, s}^{\delta}(\mathbb{R})},
\end{aligned}
$$

where

$$
\|v\|_{G_{r, s}^{\delta}(\mathbb{R})}=\left\|\Lambda^{-2}\left(\gamma_{x}+\frac{\gamma^{2}}{2 \lambda}\right)\right\|_{G_{r, s}^{\delta}(\mathbb{R})} \leq \frac{1}{2}\|\gamma\|_{G_{r, s}^{\delta}(\mathbb{R})}+\frac{C_{s}}{2}\|\gamma\|_{G_{r, s}^{\delta}(\mathbb{R})}^{2} .
$$

Combining (3.9) and (3.10), we can get

$$
\begin{aligned}
\|F(\gamma)\|_{G_{r, s}^{\delta}(\mathbb{R})} \leq & 2 C_{s}\|\gamma\|_{G_{r s}^{\delta}(\mathbb{R})}^{2}+\frac{C_{s}}{2}\|\gamma\|_{G_{r, s}^{\delta}(\mathbb{R})}+\frac{C_{s} e^{-r} r^{r}}{2\left(\delta-\delta^{\prime}\right)^{r}}\|\gamma\|_{G_{r, s}^{\delta}(\mathbb{R})}^{2} \\
& +\frac{C_{s}\left(e^{-r} r^{r}+2\right)}{2\left(\delta-\delta^{\prime}\right)^{r}}\|\gamma\|_{G_{r, s}^{\delta}(\mathbb{R})}^{3} \\
\leq & \frac{C_{s}\left(e^{-r} r^{r}+2\right)\left(\|\gamma\|_{G_{r s}^{\delta}(\mathbb{R})}^{2}+\|\gamma\|_{G_{r, s}^{\delta}(\mathbb{R})}^{3}\right)+C_{s}\|\gamma\|_{G_{r, s}^{\delta}(\mathbb{R})}}{2\left(\delta-\delta^{\prime}\right)^{r}} .
\end{aligned}
$$

Thus condition (1) in Theorem 1.1 holds.

According to the same token as the above, we can get that

$$
\left\|F\left(\gamma_{0}\right)\right\|_{G_{r, s}^{\delta}(\mathbb{R})} \leq \frac{C_{s}\left(e^{-r} r^{r}+2\right)\left(\left\|\gamma_{0}\right\|_{G_{r, s}^{\delta}(\mathbb{R})}^{2}+\left\|\gamma_{0}\right\|_{G_{r, s}^{\delta}(\mathbb{R})}^{3}\right)+C_{s}\left\|\gamma_{0}\right\|_{G_{r, s}^{\delta}(\mathbb{R})}}{2\left(\delta-\delta^{\prime}\right)^{r}} .
$$

So we see that $F(\gamma)$ satisfies the condition (3) in Theorem 2.1 with

$$
M=\frac{C_{s}\left(e^{-r} r^{r}+2\right)\left(\left\|\gamma_{0}\right\|_{G_{r, s}^{\delta}(\mathbb{R})}^{2}+\left\|\gamma_{0}\right\|_{G_{r, s}^{\delta}(\mathbb{R})}^{3}\right)+C_{s}\left\|\gamma_{0}\right\|_{G_{r, s}^{\delta}(\mathbb{R})}}{2} .
$$

Now, we only need to prove that $F(\gamma)$ satisfies the condition (2) in Theorem 2.1. Suppose that $\left\|\gamma_{1}-\gamma_{0}\right\|_{G_{r, s}^{s}(\mathbb{R})} \leq R$ and $\left\|\gamma_{2}-\gamma_{0}\right\|_{G_{r, s}^{s}(\mathbb{R})} \leq R$, applying the estimates (2.4)-(2.7), we can get 


$$
\begin{aligned}
& \left\|F\left(\gamma_{1}\right)-F\left(\gamma_{2}\right)\right\|_{G_{r, s}^{\delta^{\prime}}(\mathbb{R})}=\left\|\frac{\gamma_{1}^{2}}{2}+\lambda v_{1}-\left(v_{1} \gamma_{1}\right)_{x}-\left(\frac{\gamma_{2}^{2}}{2}+\lambda v_{2}-\left(v_{2} \gamma_{2}\right)_{x}\right)\right\|_{G_{r, s}^{\delta^{\prime}}(\mathbb{R})} \\
& \leq \frac{C_{s}\left(e^{-r} r^{r}+2\right)}{\left(\delta-\delta^{\prime}\right)^{r}}\left(\left\|\gamma_{1}\right\|_{G_{r, s}^{\delta}(\mathbb{R})}+\left\|\gamma_{2}\right\|_{G_{r, s}^{\delta}(\mathbb{R})}\right)\left\|\gamma_{1}-\gamma_{2}\right\|_{G_{r, s}^{\delta}(\mathbb{R})} \\
& \quad+\frac{C_{s}\left(e^{-r} r^{r}+2\right)}{\left(\delta-\delta^{\prime}\right)^{r}}\left(\left\|\gamma_{1}\right\|_{G_{r, s}^{\delta}(\mathbb{R})}\left\|\gamma_{1}+\gamma_{2}\right\|_{G_{r, s}^{\delta}(\mathbb{R})}\right)\left\|\gamma_{1}-\gamma_{2}\right\|_{G_{r, s}^{\delta}(\mathbb{R})} \\
& \quad+\frac{C_{s}\left(e^{-r} r^{r}+2\right)}{\left(\delta-\delta^{\prime}\right)^{r}}\left(\left\|\gamma_{2}\right\|_{G_{r, s}^{\delta}(\mathbb{R})}^{2}+1\right)\left\|\gamma_{1}-\gamma_{2}\right\|_{G_{r, s}^{\delta}(\mathbb{R})} \\
& \leq \frac{3 C_{s}\left(e^{-r} r^{r}+2\right)}{\left(\delta-\delta^{\prime}\right)^{r}}\left[\left(\left\|\gamma_{0}\right\|_{G_{r, s}^{\delta}(\mathbb{R})}+R+1\right)\left(\left\|\gamma_{0}\right\|_{G_{r, s}^{\delta}(\mathbb{R})}+R\right)+\frac{1}{3}\right]\left\|\gamma_{1}-\gamma_{2}\right\|_{G_{r, s}^{\delta}(\mathbb{R})} .
\end{aligned}
$$

According to the above inequality, we have proved that $F(\gamma)$ satisfies the condition

(2) of Theorem 2.1 with $L=3 C_{s}\left(e^{-r} r^{r}+2\right)\left[\left(\left\|\gamma_{0}\right\|_{G_{r, s}^{\delta}(\mathbb{R})}+R+1\right)\left(\left\|\gamma_{0}\right\|_{G_{r, s}^{\delta}(\mathbb{R})}+R\right)+\frac{1}{3}\right], \quad$ and that $T_{0}=\min \left\{\frac{1}{2^{2 r+4} L}, \frac{\left(2^{r+1}\right) R}{\left(2^{r+1}\right) 2^{2 r+3} L R+M D_{r}}\right\}$, by setting $R=\left\|\gamma_{0}\right\|_{G_{r, s}^{1}(\mathbb{R})}$, we can obtain that

$$
L=12 C_{s}\left(e^{-r} r^{r}+2\right)\left[\left(\left\|\gamma_{0}\right\|_{G_{r, s}^{1}(\mathbb{R})}+\frac{1}{2}\right)\left\|\gamma_{0}\right\|_{G_{r, s}^{1}(\mathbb{R})}+\frac{1}{12}\right]
$$

and $M \leq \frac{2^{2 r+3}\left(2^{r+1}\right) L R}{D_{r}}$. Thus, we get $T_{0}=\frac{1}{C_{s} 2^{2 r+7}\left(e^{-r} r^{r}+2\right)\left[\left(\left\|\gamma_{0}\right\|_{G_{r, s}^{1}(\mathbb{R})}+\frac{1}{2}\right)\left\|\gamma_{0}\right\|_{G_{r, s}^{1}(\mathbb{R})}+\frac{1}{12}\right]}$.

Next, we will prove the Theorem 1.2 as follows.

Proof of Theorem 1.2 Applying the proof of Theorem 1.1, for the given $r \geq 1$, $s>1 / 2$, we can know that the lifespan of the corresponding solution to the $\mathrm{mCH}$ Cauchy problem $\gamma^{n}$ and $\gamma^{\infty}$ are given by

$$
\begin{gathered}
T^{n}=\frac{1}{C_{s} 2^{2 r+7}\left(e^{-r} r^{r}+2\right)\left[\left(\left\|\gamma_{0}^{n}\right\|_{G_{r, s}^{1}(\mathbb{R})}+\frac{1}{2}\right)\left\|\gamma_{0}^{n}\right\|_{G_{r, s}^{1}(\mathbb{R})}+\frac{1}{12}\right]}, \\
T^{\infty}=\frac{1}{C_{s} 2^{2 r+7}\left(e^{-r} r^{r}+2\right)\left[\left(\left\|\gamma_{0}^{\infty}\right\|_{G_{r, s}^{1}(\mathbb{R})}+\frac{1}{2}\right)\left\|\gamma_{0}^{\infty}\right\|_{G_{r, s}^{1}(\mathbb{R})}+\frac{1}{12}\right]} .
\end{gathered}
$$

Since $\left\|\gamma_{0}^{n}-\gamma_{0}^{\infty}\right\|_{G_{r, s}^{1}(\mathbb{R})} \rightarrow 0$, thus, there exists a constant $N$ such that, if $n \geq N$, we get

$$
\left\|\gamma_{0}^{n}\right\|_{G_{r, s}^{1}(\mathbb{R})} \leq\left\|\gamma_{0}^{\infty}\right\|_{G_{r, s}^{1}(\mathbb{R})}+1
$$

So for $n \geq N$, we can infer that

$$
\begin{aligned}
T & \doteq \frac{1}{C_{s} 2^{2 r+7}\left(e^{-r} r^{r}+2\right)\left[\left(\left\|\gamma_{0}^{\infty}\right\|_{G_{r, s}^{1}(\mathbb{R})}+\frac{3}{2}\right)\left(\left\|\gamma_{0}^{\infty}\right\|_{G_{r, s}^{1}(\mathbb{R})}+1\right)+\frac{1}{12}\right]} \\
& \leq \min \left\{T^{n}, T^{\infty}\right\} .
\end{aligned}
$$


As in proof Theorem 1.1, we see that $T^{n}$ and $T^{\infty}$ are the existence time corresponding to $\left\|\gamma_{0}^{n}\right\|_{G_{r, s}^{1}(\mathbb{R})}$ and $\left\|\gamma_{0}^{\infty}\right\|_{G_{r, s}^{1}(\mathbb{R})}$ respectively. Which implies that for any $n \geq N$

$$
\begin{array}{cl}
\gamma^{n}(t, x)=\gamma_{0}^{n}(x)+\int_{0}^{t} F\left(\gamma^{n}(t, \tau)\right) d \tau, & 0 \leq t \leq D_{r} \frac{T(1-\delta)^{r}}{2^{r+1}}, \\
\gamma^{\infty}(t, x)=\gamma_{0}^{\infty}(x)+\int_{0}^{t} F\left(\gamma^{\infty}(t, \tau)\right) d \tau, & 0 \leq t \leq D_{r} \frac{T(1-\delta)^{r}}{2^{r+1}},
\end{array}
$$

where $F$ is given in (3.8). On the basis of the above equation, we affirm that for any $0 \leq t<D_{r} \frac{T(1-\delta)^{r}}{2^{r+1}}$ and $0<\delta<1$,

$$
\begin{aligned}
& \left\|\gamma^{n}(t)-\gamma^{\infty}(t)\right\|_{G_{r, s}^{\delta}(\mathbb{R})} \leq\left\|\gamma_{0}^{n}-\gamma_{0}^{\infty}\right\|_{G_{r, s}^{\delta}(\mathbb{R})} \\
& +\int_{0}^{t}\left\|F\left(\gamma^{n}(\tau)\right)-F\left(\gamma^{\infty}(\tau)\right)\right\|_{G_{r, s}^{\delta}(\mathbb{R})} d \tau .
\end{aligned}
$$

In consideration of (3.11), we have $\left\|F\left(\gamma^{n}(\tau)\right)-F\left(\gamma^{\infty}(\tau)\right)\right\|_{G_{r, s}^{\delta}(\mathbb{R})} \leq \frac{L\left\|\gamma^{n}-\gamma^{\infty}\right\|_{G_{r, s}^{\delta(\tau)}(\mathbb{R})}}{(\delta(\tau)-\delta)^{r}}$ which $L=12 C_{s}\left(e^{-r} r^{r}+2\right)\left[\left(\left\|\gamma_{0}\right\|_{G_{r, s}^{1}(\mathbb{R})}+\frac{1}{2}\right)\left\|\gamma_{0}\right\|_{G_{r, s}^{1}(\mathbb{R})}+\frac{1}{12}\right]^{r, s}$ with $\delta<\delta(\tau)<1$ to be confirmed later. Plugging it into (3.18) obtains that

$$
\left\|\gamma^{n}(t)-\gamma^{\infty}(t)\right\|_{G_{r, s}^{\delta}(\mathbb{R})} \leq\left\|\gamma_{0}^{n}-\gamma_{0}^{\infty}\right\|_{G_{r, s}^{\delta}(\mathbb{R})}+L \int_{0}^{t} \frac{\left\|\gamma^{n}-\gamma^{\infty}\right\|_{G_{r, s}^{\delta(\tau)}(\mathbb{R})}}{(\delta(\tau)-\delta)^{r}}
$$

Define that $\delta(\tau)=\frac{1+\delta}{2}+\left(\frac{1}{2}\right)^{2+\frac{1}{r}}\left\{\left[(1-\delta)^{r}-\frac{\tau}{T}\right]^{\frac{1}{r}}-\left[(1-\delta)^{r}+\left(2^{r+1}-1\right) \frac{\tau}{T}\right]^{\frac{1}{r}}\right\}$, using the Lemma 3.7 in [22] with $a=T$, we derive that

$$
\left\|\gamma^{n}(t)-\gamma^{\infty}(t)\right\|_{G_{r, s}^{\delta}(\mathbb{R})} \leq\left\|\gamma_{0}^{n}-\gamma_{0}^{\infty}\right\|_{G_{r, s}^{\delta}(\mathbb{R})}+\frac{2^{2 r+3} L T\left\|\gamma^{n}-\gamma^{\infty}\right\|_{E_{T}}}{(1-\delta)^{r}} \sqrt{\frac{T(1-\delta)^{r}}{T(1-\delta)^{r}-t}} .
$$

According to the definition of $L$ and $T$ (see (3.12) and (3.15)), we can obtain that $2^{2 r+3} L T<\frac{1}{2}$. So we get

$$
\begin{gathered}
\left\|\gamma^{n}(t)-\gamma^{\infty}(t)\right\|_{G_{r, s}^{\delta}(\mathbb{R})} \leq\left\|\gamma_{0}^{n}-\gamma_{0}^{\infty}\right\|_{G_{r, s}^{\delta}(\mathbb{R})} \\
+\frac{\left\|\gamma^{n}-\gamma^{\infty}\right\|_{E_{T}}}{2(1-\delta)^{r}} \sqrt{\frac{T(1-\delta)^{r}}{T(1-\delta)^{r}-t}}
\end{gathered}
$$

which leads to 


$$
\begin{aligned}
(1 & -\delta)^{r} \sqrt{1-\frac{t}{T(1-\delta)^{r}}}\left\|\gamma^{n}(t)-\gamma^{\infty}(t)\right\|_{G_{r, s}^{\delta}(\mathbb{R})} \\
& \leq(1-\delta)^{r}\left\|\gamma_{0}^{n}-\gamma_{0}^{\infty}\right\|_{G_{r, s}^{\delta}(\mathbb{R})} \sqrt{1-\frac{t}{T(1-\delta)^{r}}}+\frac{1}{2}\left\|\gamma^{n}-\gamma^{\infty}\right\|_{E_{T}} \\
& \leq\left\|\gamma_{0}^{n}-\gamma_{0}^{\infty}\right\|_{G_{r, s}^{1}(\mathbb{R})}+\frac{1}{2}\left\|\gamma^{n}-\gamma^{\infty}\right\|_{E_{T}} .
\end{aligned}
$$

We can find easily that the right term of the above posture is independence of $t$ and $\delta$. In the light of the definition of $E_{T}$, we get

$$
\begin{aligned}
& \left\|\gamma^{n}(t)-\gamma^{\infty}(t)\right\|_{E_{T}} \\
& \quad=\sup _{|t|<D_{r} \frac{T(1-\delta)^{r}}{2^{r+1}}, 0<\delta<1}(1-\delta)^{r} \| \gamma^{n}(t)-\gamma^{\infty}(t) \\
& \quad \|_{G_{r, s}^{\delta}(\mathbb{R})} \sqrt{1-\frac{t}{T(1-\delta)^{r}}} \\
& \quad \leq\left\|\gamma_{0}^{n}-\gamma_{0}^{\infty}\right\|_{G_{r, s}^{1}(\mathbb{R})} .
\end{aligned}
$$

The above inequality holds true for any $n \geq N$, and leads to our desire results.

\section{Conclusions}

This paper mainly studies the Cauchy problem of the modified Camassa-Holm equation. According to the Ovsyannikov theorem and the basic properties of SobolevGevrey spaces, we prove the Gevrey regularity and analyticity of the equation (see Theorem 1.1). In addition, we obtain a lower bound of the lifespan and the continuity of the data-to-solution map (see Theorem 2.2).

Abbreviations For any $s \in \mathbb{R}$ we take the operator $\Lambda^{s}=\left(1-\partial_{x}^{2}\right)^{s / 2}$ to be defined by $\widehat{\Lambda^{s}} f(\xi)=\left(1+\xi^{2}\right)^{s / 2} \hat{f}(\xi)$, where $\hat{f}(\xi)$ is the Fourier transform, $\hat{f}(\xi) \doteq \int_{\mathbb{R}} e^{-i x \xi} f(x) d x$. Then, for $f \in H^{s}(\mathbb{T})$ we have

$$
\|f\|_{H^{s}} \doteq\|f\|_{H^{s}(\mathbb{R})}=\left(\int_{\mathbb{R}}\left(1+\xi^{2}\right)^{s}|\hat{f}(\xi)|^{2} d \xi\right)^{1 / 2}<\infty .
$$

Acknowledgements The author would like to thank anonymous reviewers and editors for their careful reading and valuable suggestions, which greatly improved the quality of the paper.

Author Contributions Shouming Zhou proposed the problem that what is the gevrey regularity and analyticity for the modified Camassa-Holm Equation; Qingyu Zhou studied this problem and written the original manuscript; Xiaolin Pan revised the manuscript and proved Theorem 1.1.

Funding This work is supported by Natural Science Foundation of Chongqing (Grant No.csts2020jcyjjqX0022) and the Chongqing's youth talent support program (Grant No. cstc2021ycjh-bgzxm0130), 
Science and Technology Research Program of Chongqing Municipal Educational Commission (Grant Nos. KJZD-M201900501, KJQN202000518).

Availability of data and material Not applicable.

\section{Declarations}

Conflict of interest The authors declare that they have no conflicts of interest.

Ethics approval and consent to participate Not applicable.

Consent for publication Not applicable.

Open Access This article is licensed under a Creative Commons Attribution 4.0 International License, which permits use, sharing, adaptation, distribution and reproduction in any medium or format, as long as you give appropriate credit to the original author(s) and the source, provide a link to the Creative Commons licence, and indicate if changes were made. The images or other third party material in this article are included in the article's Creative Commons licence, unless indicated otherwise in a credit line to the material. If material is not included in the article's Creative Commons licence and your intended use is not permitted by statutory regulation or exceeds the permitted use, you will need to obtain permission directly from the copyright holder. To view a copy of this licence, visit http://creativecommons.org/licen ses/by/4.0/.

\section{References}

1. Camassa, R., Holm, D.: An integrable shallow water equation with peaked solitons. Phys. Rev. Lett. 71, 1661-1664 (1993)

2. Camassa, R., Holm, D.D., Hyman, J.: A new integrable shallow water equation. Adv. Appl. Mech. 31, 1-33 (1994)

3. Constantin, A.: The Hamiltonian structure of the Camassa-Holm equation. Exposit. Math. 15, 53-85 (1997)

4. Constantin, A.: On the scattering problem for the Camassa-Holm equation. R. Soc. Lond. Proc. Ser. A Math. Phys. Eng. Sci. 457, 953-970 (2001)

5. Constantin, A.: The trajectories of particles in Stokes waves. Invent. Math. 166, 523-535 (2006)

6. Constantin, A.: Particle trajectories in extreme Stokes waves. IMA J. Appl. Math. 77, 293-307 (2012)

7. Constantin, A., Escher, J.: Well-posedness, global existence, and blowup phenomena for a periodic quasi-linear hyperbolic equation. Commun. Pure Appl. Math. 51, 475-504 (1998)

8. Constantin, A., Molinet, L.: Global weak solutions for a shallow water equation. Commun. Math. Phys. 211, 45-61 (2000)

9. Constantin, A., Strauss, W.A.: Stability of peakons. Commun. Pure Appl. Math. 53, 603-610 (2000)

10. Constantin, A., Strauss, W.A.: Stability of the Camassa-Holm solitons. J. Nonlinear. Sci. 12, 415422 (2002)

11. Constantin, A., Gerdjikov, V.S., Ivanov, R.I.: Inverse scattering transform for the Camassa-Holm equation. Inverse Probl. 22, 2197-2207 (2006)

12. Eckhardt, J.: The inverse spectral transform for the conservative Camassa-Holm flow with decaying initial data. Arch. Ration. Mech. Anal. 224, 21-52 (2017)

13. Foias, C., Temam, R.: Gevrey class regularity for the solutions of the Navier-Stokes equations. J. Funct. Anal. 87, 359-369 (1989)

14. Fokas, A.S., Fuchssteiner, B.: Symplectic structures, their Bäklund transformations and hereditary symmetries. Phys. D 4, 47-66 (1981)

15. Górka, P., Reyes, E.G.: The modified Camassa-Holm equation. Int. Math. Res. Not. 12, 2617-2649 (2011) 
16. Hernández, H.R., Reyes, E.G.: Geometric integrability of the Camassa-Holm equation. Int. Math. Res. Not. 13, 3089-3125 (2012)

17. Himonas, A.A., Misiolek, G.: Analyticity of the Cauchy problem for an integrable evolution equation. Math. Ann. 327, 575-584 (2003)

18. Luo, W., Yin, Z.: Gevrey regularity and analyticity for Camassa-Holm type systems. Ann. Sc. Norm. Super. Pisa Cl. Sci. 18, 1061-1079 (2018)

19. Luo, Z., Qiao, Z., Yin, Z.: On the Cauchy problem for a modified Camassa-Holm equation. Monatsh. Math. 193, 857-877 (2020)

20. Luo, Z., Qiao, Z., Yin, Z.: Globally conservative solutions for the modified Camassa-Holm (MOCH) equation. J. Math. Phys. 62, 091506 (2021)

21. Reyes, E.G.: Geometric integrability of the Camassa-Holm equation. Lett. Math. Phys. 59, 117-131 (2002)

22. Zhang, L., Liu, B.: On the Luo-Yin results concerning Gevrey regularity and analyticity for Camassa-Holm-type systems. Ann. Sci. Norm. Super. Pisa Cl. Sci. 21, 1741-1744 (2020) 